\title{
COHERENCE OF POLYNOMIAL RINGS OVER SEMISIMPLE ALGEBRAIC ALGEBRAS
}

\author{
ANDREW B. CARSON ${ }^{1}$
}

\begin{abstract}
It is shown that polynomial rings in finitely or infinitely many central indeterminates, over a commutative algebraic algebra without nilpotent elements, are coherent. If the coefficient ring is algebraic over the real numbers, then the commutativity assumption, above, may be dropped.
\end{abstract}

In this paper all rings have identity, all modules are unital, and all ring homomorphisms preserve the identity.

Definition 1. A ring $R$ is left coherent if, for each finitely generated left ideal $I$ in $R$, there exists an exact sequence of left $R$-modules

$$
0 \rightarrow K \rightarrow F \rightarrow I \rightarrow 0
$$

such that $F$ and $K$ are finitely generated and $F$ is free.

Right coherent rings may be similarly defined. The concept of a coherent ring was introduced by Chase in [4]. He showed, in [4, Theorem 2.1], that a ring $R$ is left coherent if and only if the direct product of any family of flat right $R$-modules is flat. As left Noetherian rings are clearly left coherent, this suggests that left coherent rings are, at least with respect to homological properties, a generalization of left Noetherian rings. This raises the following question: If $R$ is an arbitrary left coherent ring, is the polynomial ring $R[Z]$ left coherent too? Soublin, in [10], answered this question in the negative, even for commutative $R$. However, he showed in [9, Theorems 21 and 22] that if $R$ is commutative and von Neumann regular (i.e. for each $r \in R$ there exists $r^{\prime} \in R$ such that $r r^{\prime} r=r$ ), then $R[Z]$ is coherent and its finitely generated ideals are principal.

In this paper we prove the following result:

THEOREM. Let $A$ be a central algebraic algebra, without nilpotent elements, over some field L. Suppose that at least one of the following two

Received by the editors August 6, 1971 .

AMS 1969 subject classifications. Primary 1320, 1620; Secondary 1656, 1644.

Key words and phrases. Commutative semisimple algebraic algebras, polynomial rings, coherent rings, topological representations, faithfully flat over rings, direct limits of coherent rings.

${ }^{1}$ The author was supported at the University of Hawaii by a National Research Council of Canada Postdoctorate fellowship while carrying out research for this paper.

(c) American Mathematical Society 1972 
hypothesis is satisfied:

( $\alpha) A$ is commutative,

( $\beta) L$ is the field of real numbers.

Then the polynomial ring $A\left[\left\{Z_{\alpha}\right\}\right]$ is left and right coherent, for any finite or infinite set $\left\{Z_{\alpha}\right\}$ of central indeterminates.

The above meanings for $A, L$, and $\left\{Z_{\alpha}\right\}$ are retained throughout this paper. By [1, Theorems 3.2 and 3.3], $A$ is von Neumann regular. The proof of the above theorem requires the following lemmas and propositions.

Proposition 1. Let $R$ be a ring. For $r \in R$ let $(0: r)$ denote $\{s \in R: s r=0\}$. Then $R$ is left coherent if and only if

(i) for each $r \in R,(0: r)$ is finitely generated as a left ideal in $R$,

(ii) the intersection of any two finitely generated left ideals in $R$ is again finitely generated.

Proposition 2. Let $\left\{R_{\alpha}\right\}$ be a directed system of left coherent rings such that, when $\alpha \leqq \beta, R_{\beta}$ is flat as a right $R_{\alpha}$-module. Then the direct limit of $\left\{R_{\alpha}\right\}$ is a left coherent ring.

Definition 2. Let $R$ be a subring of the ring $S$. Then $S$ is faithfully right flat over $R$ if

(i) $S$ is flat as a right $R$-module,

(ii) if $M$ is a left $R$-module such that $S \otimes_{R} M=0$, then $M=0$.

Proposition 3. Let $R$ be a subring of the left coherent ring $S$, such that $S$ is faithfully right flat over $R$. Then $R$ is a left coherent ring.

Proposition 1 is part of [4, Theorem 2.2]. Proposition 2 is from [2, p. 63, Example 12]. Proposition 3 is [5, Corollary 2.1].

Notation. (i) We recall that a topological space is Boolean if it is compact, Hausdorff, and totally disconnected. A subset of a topological space $X$ is clopen if it is both open and closed in $X$.

(ii) For any ring $R$ and Boolean space $X$, let $\mathscr{C}(X, R)$ denote the ring of all continuous functions from $X$ to $R$, where $R$ has the discrete topology. In other words, $\mathscr{C}(X, R)$ is the ring of all locally constant functions from $X$ to $R$. For $f \in \mathscr{C}(X, R)$ let $\operatorname{ker}(f)$ denote $\{x \in X: f(x)=0\}$. Clearly $\operatorname{ker}(f)$ is clopen in $X$.

(iii) If $I$ is a left ideal in $\mathscr{C}(X, R)$ and $x \in X$, let $I_{x}$ denote $\{f(x): f \in I\}$. Clearly $I_{x}$ is a left ideal in $R$. It is easy to see, where $J$ is also a left ideal in $\mathscr{C}(X, R)$, that $(I \cap J)_{x}=I_{x} \cap J_{x}$.

(iv) If $A$ is commutative let $F$ denote the algebraic closure of $L$. Otherwise let $F$ denote the real quaternions.

Our results hinge upon the following topological representation of $A$. 
Proposition 4. There is an embedding

$$
A \rightarrow \mathscr{C}(X, F),
$$

for some Boolean space $X$.

Proof. If $A$ is commutative this is contained in [1, Theorem 6.1]. An alternate proof, if $A$ is commutative, and the noncommutative case, occur in [3, Theorem 3.4 and concluding remark (d)].

Notation. Let $B$ denote the ring $\mathscr{C}(X, F)$ from Proposition 4 . Let $p \geqq 1$ be an integer. Let $T$ denote the polynomial ring $F\left[Z_{1}, \cdots, Z_{p}\right]$. Clearly

$$
B\left[Z_{1}, \cdots, Z_{p}\right] \cong \mathscr{C}(X, T) .
$$

LeMma 1. The ring $B\left[Z_{1}, \cdots, Z_{p}\right]$ is left coherent.

Proof. We shall use Proposition 1 to show that $\mathscr{C}(X, T)$ is left coherent.

First let $f$ be an arbitrary element in $\mathscr{C}(X, T)$. Define a map $e: X \rightarrow T$ by $e(x)=1$ when $x \in \operatorname{ker}(f)$ and $e(x)=0$ when $x \notin \operatorname{ker}(f)$. The map $e$ is continuous since $\operatorname{ker}(f)$ is clopen in $X$. Clearly $e$ generates $(0: f)$.

Second, let $I$ and $J$ be two finitely generated left ideals in $\mathscr{C}(X, T)$. Suppose that $x \in X$. Since elements of $\mathscr{C}(X, T)$ are locally constant functions, there exists a neighborhood $N_{x}$ of $x$ such that, for $y \in N_{x}, I_{y}=I_{x}$ and $J_{y}=J_{x}$. Since $T$ is left Noetherian, $I_{x} \cap J_{x}$ can be generated, for some integer $n(x) \geqq 1$, by elements $t_{1}(x), \cdots, t_{n(x)}(x)$ of $T$. Since $\left\{N_{x}: x \in X\right\}$ is an open cover of the Boolean space $X$, there is for some integer $m \geqq 1$, by [8, p. 12], a family $\left\{V_{j}: 1 \leqq j \leqq m\right\}$ of clopen subsets of $X$ such that

(a) $\bigcup_{j=1}^{m}\left(V_{j}\right)=X$,

(b) $V_{i} \cap V_{j}=\varnothing$ if $i \neq j$,

(c) for each $j$, where $1 \leqq j \leqq m$, there exists $x(j) \in X$ such that $V_{j} \subseteq N_{x(j)}$. Let $n=\sup \{n(x(j)): 1 \leqq j \leqq m\}$. Whenever $n(x(j))<i \leqq n$ set $t_{i}(x(j))=0$. For each $i$, where $1 \leqq i \leqq n$, define the map $h_{i}: X \rightarrow T$ by $h_{i}(x)=t_{i}(x(j))$ where $x \in V_{j}$. In view of (a)-(c) the $h_{i}$ are well defined. They are in $\mathscr{C}(X, T)$ since the $V_{j}$ are clopen. Let $H$ be the ideal in $\mathscr{C}(X, T)$ generated by $\left\{h_{i}: 1 \leqq i \leqq n\right\}$. By construction $(I \cap J)_{x}=I_{x} \cap J_{x}=H_{x}$ for each $x \in X$. This establishes, via a compactness argument similar to the one given above, that $I \cap J=H$. Hence $I \cap J$ is finitely generated. The lemma now follows from Proposition 1.

In view of Proposition 3 we could now establish that $A\left[Z_{1}, \cdots, Z_{p}\right]$ is left coherent by showing that $B\left[Z_{1}, \cdots, Z_{p}\right]$ is faithfully right flat over $A\left[Z_{1}, \cdots, Z_{p}\right]$.

LEMMA 2. The ring $B$ is faithfully right flat over $A$. 
Proof. It is established in [1, Theorems 3.2 and 3.3] that $A$ is a von Neumann regular ring. By [7, Proposition 4], such rings have the property that all of their modules, left and right, are flat. In particular, $B$ is flat as a right $A$-module.

Let $M$ be a left $A$-module. Suppose that $B \otimes_{A} M=0$. Then, using the flatness of $M$ as a left $A$-module, we have

Thus $M=0$.

$$
M \cong A \otimes_{A} M \subseteq B \otimes_{A} M=0 .
$$

LEMMA 3. Suppose that the ring $S$ is faithfully right flat over the subring $R$. Then $S[Z]$ is faithfully right flat over $R[Z]$.

Proof. First note that, as left $S$-modules, $S[Z] \cong S \otimes_{R} R[Z]$.

Next note that, for any left $S[Z]$-module $M$, there are the following left $S$-module isomorphisms:

$$
S[Z] \otimes_{R[Z]} M \cong S \otimes_{R} R[Z] \otimes_{R[Z]} M \cong S \otimes_{R} M .
$$

Thus, for any left $S[Z]$-modules $M$ and $N$ and homomorphism $f: M \rightarrow N$, the following diagram is commutative and its columns are isomorphisms, where $f^{\prime}$ and $f^{*}$ are natural maps induced by $f$ :

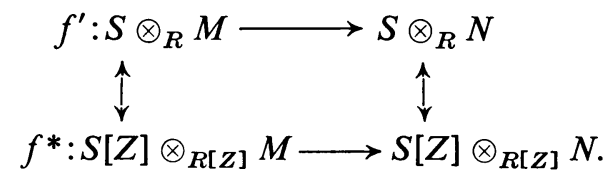

Since $S$ is faithfully right flat over $R$ it follows that if $f$ is a monomorphism, then so are $f^{\prime}$ and (by the diagram) $f^{*}$. Thus $S[Z]$ is a flat right $R[Z]$-module. Similarly, if $S[Z] \otimes_{R[Z]} M=0$, then $S \otimes_{R} M=0$ so that $M=0$.

Lemma 4. The ring $A\left[Z_{1}, \cdots, Z_{p}\right]$ is left coherent.

Proof. It follows from Lemma 2 and $p$ applications of Lemma 3, that $B\left[Z_{1}, \cdots, Z_{p}\right]$ is faithfully right flat over $A\left[Z_{1}, \cdots, Z_{p}\right]$. The result now follows from Lemma 1 and Proposition 3.

THEOREM. Let $\left\{Z_{\alpha}\right\}$ be any set (finite or infinite) of central indeterminates. Then $A\left[\left\{Z_{\alpha}\right\}\right]$ is left coherent.

PRoof. The family $\left\{A\left[Z_{1}, \cdots, Z_{n}\right]: n \geqq 1\right.$ and $\left.\left\{Z_{1}, \cdots, Z_{n}\right\} \subseteq\left\{Z_{\alpha}\right\}\right\}$ is directed under inclusion. Clearly

$$
\lim _{\rightarrow}\left(A\left[Z_{1}, \cdots, Z_{n}\right]\right) \cong A\left[\left\{Z_{\alpha}\right\}\right] .
$$

The theorem now follows from Lemma 4 and Proposition 2. 
REMARK. The definition of a nilpotent element is left-right symmetric. Thus $A\left[\left\{Z_{\alpha}\right\}\right]$ is also a right coherent ring.

COROllaRY. Suppose that $R$ is a ring such that, for each $r \in R$, there is an integer $m(r) \geqq 2$ satisfying

$$
r^{m(r)}=r .
$$

Then $R\left[\left\{Z_{\alpha}\right\}\right]$ is a commutative (left) coherent ring, for any finite or infinite set $\left\{Z_{\alpha}\right\}$ of central indeterminates.

Proof. It is a well-known result, due to Jacobson, that $R$ is commutative. As in [8, Corollary 12.5] there exists a finite set of prime integers $\left\{p_{1}, \cdots, p_{n}\right\}$ and a ring direct sum decomposition of $R$,

$$
R \cong R_{1} \oplus \cdots \oplus R_{n},
$$

such that each $R_{i}$ has characteristic $p_{i}$. Thus, as each $R_{i}$ satisfies (*), it is an algebraic algebra without nilpotent elements over the field with $p_{i}$ elements. Hence $R_{i}\left[\left\{Z_{\alpha}\right\}\right]$ is left coherent, for $1 \leqq i \leqq n$. Clearly

$$
R\left[\left\{Z_{\alpha}\right\}\right] \cong R_{1}\left[\left\{Z_{\alpha}\right\}\right] \oplus \cdots \oplus R_{n}\left[\left\{Z_{\alpha}\right\}\right]
$$

The result now follows from [6, Corollary 2.1] which states that a finite direct product of left coherent rings is again left coherent.

\section{REFERENCES}

1. R. F. Arens and I. Kaplansky, Topological representation of algebras, Trans. Amer. Math. Soc. 63 (1948), 457-481. MR 10, 7.

2. N. Bourbaki, Eléments de mathématique. Fasc. XXVII. Algèbre commutative. Chap. 1: Modules plats. Chap. 2: Localisation, Actualités Sci. Indust., no. 1290, Hermann, Paris, 1961. MR 36 \#146.

3. A. Carson, Representation of semi-simple algebraic algebras, J. Algebra (to appear).

4. S. Chase, Direct products of modules, Trans. Amer. Math. Soc. 97 (1960), 457-473. MR 22 \#11017.

5. M. Harris, Some results on coherent rings, Proc. Amer. Math. Soc. 17 (1966), 474-479. MR 33 \#1332.

6. - Some results on coherent rings. II, Glasgow Math. J. 8 (1967), 123-126. MR 36 \#6453.

7. J. Lambek, Lectures on rings and modules, Blaisdell, Waltham, Mass., 1966. MR 34 \#5857.

8. R. S. Pierce, Modules over commutative regular rings, Mem. Amer. Math. Soc. No. 70 (1967). MR 36 \#151.

9. J. Soublin, Anneaux cohérents, C. R. Acad. Sci. Paris Sér. A-B 267 (1968), A183-A186. MR 38 \#1121.

10. Un anneau cohérent dont l'anneau des polynômes n'est pas cohérent, $\mathrm{C} . \mathrm{R}$. Acad. Sci. Paris Sér. A-B 267 (1968), A241-A243, MR 38 \#1123.

Department of Mathematics, University of Washington, Seattle, Washington 98195 